\title{
Evidence of Irf4 Gene, Irf2, Irf8 Genes in an Invertebrate: The Sea Star Asterias rubens
}

\section{Michel Leclerc ${ }^{1 *}$ and Nicolas Kresdorn ${ }^{2}$}

${ }^{1}$ Department of Cell Biology, Developmental Biology, Immunology, University of Orléans, Orléans, France ${ }^{2}$ GenXPro, Frankfurt, Germany

\author{
Abstract \\ IRF4 gene, IRF2 and IRF8 genes have been studied in sea star which is the only invertebrate, to possess these \\ factors, playing a rôle in the immune adaptative immunity of $A$. rubens.
}

Keywords: IRF4 gene; Adaptative immune system; Immune cells

\section{Introduction}

IFR regulatory factors are ancient molecules conserved throughout the evolution of metazoans; they play a fundamental roles in innate and adaptative immune system [1]. To date 11 IRF family have been described in vertebrates and invertebrates [2].

Previous studies showed that in vertebrates, IRF4 was expressed in most types of immune cells, and had critical functions in B cell differenciation and immunoglobulin production [3].

Other studies on chicken found that IRF4 was mainly expressed in the bursa, bursal lymphocytes [4] and thymus [5]. Con A induced the expression of IRF4 in spleen cells [4].

As for IRF8 in mouse, it specifically bound to the expression of type I IFR and IFR-inducible MHC Class I genes. IRF2 had the same rôle and played a regulatory one in cells of the immune system. The aim of this work is the research of such factors in an invertebrate : the sea star Asterias rubens.

\section{Materials and Methods}

Sea stars were obtained from the Biology Institute (Gothenburgh University). Immunizations to HRP, genomic studies were already described [6]. After ligation of adapters for Illumina's GSII sequencing system, the cDNA was sequenced on the Illumina GSII platform sequencing. $1.100 \mathrm{bp}$ from one side of the approximately $200 \mathrm{bp}$ fragments sequences were assembled using Velvet [7].

\section{Results}

First results (a) concern significative IRF4 gene, found in immunized sea stars to HRP, when compared to mouse genome :

a) One Contig (Contig8452|m.8105) could be annotated via BLASTX to Mus musculus "Interferon regulatory factor 4" (IRF4), with an e-value of 3.2e-12. On an aligned region of 132 amino acids, 62 positive and 42 identical amino acids were found.

5'GCCAGGTGTGCATGTACCTGAGCCAGCCGTATGGAAGA

CGAGACTGCGTACTGCTCTCAATAAACTCCCTGACATCG

ATGAGGTCCACGAGAAGACGCAACTAGACATCCCTGAAC

CCTATAGAGTCTACAGACTGCTGCCCAAAAAACCATCCA

ACAGCGCAATGAAAAACCGGCTTCCGACCAGCCCAACGT

ATGGCCAGCAGGACTCTCACCACAGGAGTGGACATTCGA
TGAGTAGTTACTCCGAGCGGTACAGTCCATATGACGTGC

GACCTGGAACTTATTACACTGATGCATACGACCGGCAAA ATCATTCGAGTTACCAACAACCGAGTCATCATTGGTATG

ATCCGAGCCGTCCCGGTGTTGATTATGGCTACCAGCAAG CATACACCAACTGTTACCCAAACAGCACAGCAGCCGACC TTGACATGATCAATGACGTCATCACACGTGACATCGATG GCAACCACGGCCACCATCCCCCAAGCCACGCCCACTACC CCCCAGGGAGCG3'

Second results (b) concern IRF2 gene in immunized and nonimmunized sea stars to HRP, when compared to mouse genome.

b) One Contig (Contig13667) could be annotated via BLASTX to Mus musculus "Interferon regulatory factor 2" (IRF2_MOUSE), with an e-value of 1.3e-39. On an aligned region of 144 amino acids, 101 positive and 75 identical amino acids were found.

5'TATGTAATCCATCAGCCCCCTCCGTACGTAGAGGGGTG

AATAAGCAGCGCCTCTGTTGCATGGATATGGGGGTGGAG GGCTTATAACTGATGCGGTTGGTGAGAGCGGGGATGGAA GGCTGGAAAGATTGCTAACTCTTGACGAGGTTGGGGAAG AGTACGGAGGAAGCTCGTGTCGCATTGGGGAACTCTGAT TGCTGAATGTAACACTCGAATGCCAAACATCTGTATTCA TCTGTGATTGGCTGACTTCGTTATGCTCTTTCCAAAGCTC AGGTGAGAATTGTTGAGAAGTCGGATGATGCGTGGACGT AATACTGTCTCGAGTCTGGAGAATTCTTGATTGCACTTTG AATAAACTGGTCTTTGGCGATCTTCTGATAGTCCTACTG

*Corresponding author: Michel Leclerc, Department of Cell Biology, Developmental Biology, Immunology, University of Orléans, Orléans, France, E-mail: mleclerc45@gmail.com

Received June 10, 2016; Accepted June 13, 2016; Published June 20, 2016

Citation: Leclerc M, Kresdorn N (2016) Evidence of Irf4 Gene, Irf2, Irf8 Genes in an Invertebrate: The Sea Star Asterias rubens. J Stem Cell Res Ther 6: 340. doi: 10.4172/2157-7633.1000340

Copyright: ( 2016 Leclerc M, et al. This is an open-access article distributed under the terms of the Creative Commons Attribution License, which permits unrestricted use, distribution, and reproduction in any medium, provided the original author and source are credited. 
Citation: Leclerc M, Kresdorn N (2016) Evidence of Irf4 Gene, Irf2, Irf8 Genes in an Invertebrate: The Sea Star Asterias rubens. J Stem Cell Res Ther 6: 340. doi: 10.4172/2157-7633.1000340

Page 2 of 2

GTCTTCTTGTGTATCGTACTGCCATCACCCACAGGTTTGC

TTTTTCTCGCCTTCTTCGGAATCAACTTGTAAATCTTGTA

AGCATCGTTCCCTTTACTCTTCCCCTGATCCTTGACCTCA

AGAATGTCCGGAAGTGAATTGATTGCACACCGGAAGTTC

GCTTTCCAGATCTTGGGATTCGCCTTCGGGCAATTGGAT

ACCGGGTCGTATTTTTTTGTATGGATGGCCCAAGCACGA

AATAGCGATGCATCTTTGTCACAAGTCCAGCCGTGTCTT

GCGGCATGTTTCCAGGGTATCTTAACCAACTGTTCCTTTT

CATCCAACCACTCCAAGCCGGGTATGGTTTTCTCAACGA

TGTTACGGATGAGCCATGGTCGCAGACGCATCCTCTCGA

\section{CCGGGCGAACCGACG 3'}

Third results (c) concern IRF8 gene in immunized and nonimmunized sea stars to HRP, when compared to mouse genome.

c) One Contig (c35396_g1_i1) could be annotated via BLASTX to Mus musculus "Interferon regulatory factor 8" from the Swissprot database (IRF8_MOUSE), with an e-value of 1e-12. On an aligned region of 53 amino acids, 42 positive and 33 identical amino acids were found

5'GGTAAATTCAAGCCAGGTGTGCATGTACCTGAGCCAGC CGTATGGAAGACGAGACTGCGTACTGCTCTCAATAAACT

CCCTGACATCGATGAGGTCCACGAGAAGACGCAACTAGA CATCCCTGAACCCTATAGAGTCTACAGACTGCTGCCCAA AAAACCATCCAACAGCGCAATGAAAAACCGGCTTCCGAC CAGCCCAACGT 3'

\section{Discussion and Conclusion}

Echinoderms occupy a critical and largely unexplored phylogenetic situation from the point of view of the evolution and of studies of the sea star immune system. Scepticism occured as for the existence of the primitive sea star antibody [8]. To day it seems obvious that the discovery of IRF4 in immunized sea stars, of IRF2 and IRF8 in both immunized and non-immunized sea stars to HRP corroborate the presence of such an invertebrate primitive antibody in Asterias rubens: an Igkappa primitive antibody. In conclusion, these IRF genes control the regulation of this last one.

\section{Reference}

1. Tamura $T$, Yanai $H$, Savitsky D, Taniguchi $T$ (2008) The IRF family transcription factors in immunity and oncogenesis. Annu Rev Immunol 26: 535-584. [PubMed]

2. Huang B, Qi ZT, Xu Z, Nie P (2010) Global characterization of interferon regulatory factor (IRF) genes in vertebrates: glimpse of the diversification in evolution. BMC Immunol 5: 11-22. [PubMed]

3. Silva NSD, Simonetti G, Heise N, Klein U (2012) The diverse roles of IRF4 in late germinal center B-cell differentiation. Immun Rev 247: 73-92. [PubMed]

4. Nehyba J, Hrdlicková R, Burnside J, Bose HR (2002) A novel interferon regulatory factor (IRF), IRF-10, has a unique role in immune defense and is induced by the v-Rel oncoprotein. Mol Cell Biol. 22: 3942-3957. [PubMed]

5. Dougherty DC, Park HM, Sanders MM (2009) Interferon regulatory factors (IRFs) repress transcription of the chicken ovalbumin gene. Gene 439: 63-70. [PubMed]

6. Leclerc M, Kresdorn N, Rotter B (2013) Evidence of complement genes in the sea-star Asterias rubens. Comparisons with the sea urchin. Immunol Lett 151: 68-70. [PubMed]

7. Zerbino DR, Birney E (2008) Velvet: algorithms for de novo short read assembly using de Bruijn graphs. Genome Res 18: 821-829. [PubMed]

8. Vincent N, Osteras M, Otten P, Leclerc M (2014) A new gene in A. rubens: A sea star Ig kappa gene. Meta Gene 2: 320-322. [PubMed] 